\title{
CLOSED FORM SOLUTIONS OF HEAT AND MASS TRANSFER IN THE FLOW OF A MHD VISCO-ELASTIC FLUID OVER A POROUS STRETCHING SHEET
}

\author{
K. ASHOKKUNAR and P.H. VEENA \\ Dept. of Mathematics, Smt. V.G. College for Women \\ Gulbarga, Karnataka, INDIA \\ E-mail: drveenaph@yahoo.com \\ V.K. PRAVIN \\ Dept. of Mech. Engg., P.D.A. College of Eng. \\ Gulbarga, Karnataka, INDIA
}

\begin{abstract}
The problem of heat and mass transfer in a visco-elastic fluid flow over a stretching sheet in the presence of a uniform magnetic field is examined. The important physical quantities such as the skin friction coefficient, heat transfer co-efficient and the mass transfer co-efficient are determined. It is found that the heat and mass transfer distribution decreases with the increasing values of the visco-elastic parameter $k_{l}$.
\end{abstract}

Key words: heat and mass transfer, MHD flow, porous stretching sheet, visco-elastic fluid.

\section{Introduction}

The flow and heat transfer on a moving solid boundary ( viz. a polymer sheet or filament ) extruded continuously from a die or a long thread traveling between a feed roll and a wind up roll in a fluid have many applications. Studies have been made by many researchers on Newtonian fluids considering various aspects such as the effect of mass transfer, wall temperature, magnetic field, porous medium etc. The heat transfer and mass transfer for the visco-elastic fluid is found to be smaller as compared with that of a Newtonian fluid. The power required in stretching the sheet in a visco-elastic fluid is smaller than when it is placed in a viscous fluid. At present visco-elastic fluids have gained considerable importance due to their extensive use in polymer industries and other industries. A visco-elastic boundary layer flow past a stretching sheet has been the subject of a large number of publications (Crane, 1970; Sakiadis, 1964; Tsou et al., 1967; Rajagopal et al., 1987; Siddeshwar and Mahabaleshwar, 2005; Chaim, 1982; Takhar and Soundalgekar, 1986; Takhar and Gorla, 1991) and also a great deal of interest has focused on the knowledge of the rheological effects of non-Newtonian (visco-elastic) fluids on the steady and un-steady flows through porous media.

Siddappa and Subhas (1985) and Rajgopal et al. (1984) studied the problem of a visco-elastic fluid flow over a stretching sheet. The uniqueness of the solution of the problem for Newtonian (viscous) fluids was examined by McLeod and Rajagopal (1987) and Troy et al. (1987). Weng-Dong Chang (1989) obtained another closed form solution for the non-Newtonian parameter $k=1 / 2$. There are many extensions of this problem which include consideration of more general stretching velocity. The situation where suction and blowing exist at the moving surface is discussed by Gupta and Gupta (1977), and Chen and Char (1986). Surmadevi and Nath (1990) solved the problem numerically for the small non-Newtonian parameter, whereas Ahmed et al. (1990) obtained the solution for large Prandtl numbers and found that the heat transfer co-efficient has no significant effect on the to non-Newtonian parameter. 
Lawrence et al. (1992) studied the heat transfer in the flow of a visco-elastic fluid over a stretching sheet. Lawrence et al. (1993) also re-investigated the study of non-uniqueness of the flow of a visco-elastic fluid over a stretching sheet.

All the above investigations are restricted to flow behavior and only heat transfer study in the absence of porous media and a magnetic field. Further, flow through porous media has gained attention and an analysis has been carried out by Abel and Veena (1998) of a non-Newtonian flow through a porous medium over an impermeable stretching surface. Singh et al. (2000) studied the effect of a transverse magnetic field on a flat plate thermometer. Layek et al. (2007) mode a heat and mass transfer analysis for a boundary layer stagnation point flow towards a heated porous sheet with heat absorption and suction or blowing. Veena et al. (2007) studied the unsteady mass transfer flow of a visco-elastic fluid over a stretching sheet saturated in a porous medium with suction or blowing. Rajgopal et al. (2008) worked on the study of diffusion of chemically reactive species of MHD visco-elastic fluid immersed in a porous medium with PST and PHF cases.

Thus motivated by all the above analyses, our present work envisages to study the visco-elastic second order fluid flow past a stretching sheet with a uniform magnetic field immersed in a saturated porous medium with both heat and mass transfer studies.

\section{Flow analysis}

An incompressible second order fluid has a constitutive equation based on the postulate of gradually fading memory given by Fosdick and Rajgopal (1979) as

$$
T=-p I+\mu A 1+\alpha_{1} A_{2}+\alpha_{2} A_{1}^{2}
$$

where $T$ is the stress tensor, $p$ is the pressure, $\mu, \alpha_{1}, \alpha_{2}$ are material constants with $\alpha_{1}<0$ and $A_{1}$ and $A_{2}$ are defined as

$$
\begin{aligned}
& A_{1}=(\operatorname{grad} v)+(\operatorname{grad} v)^{T}, \\
& A_{2}=\frac{d}{d t} A_{1}+A_{1} \cdot \operatorname{grad} v+(\operatorname{grad} v)^{T} \cdot A_{1} .
\end{aligned}
$$

Dunn and Rajgopal (1995) showed that the model (A) displays a normal stress difference in shear flow and is an approximation to a simple fluid in the sense of retardation. This model is applicable to some dilute polymer solutions and is valid at low rates of shear.

We consider heat and mass transfer of a fluid obeying the model (A) past a porous stretching sheet coinciding with the plane $y=0$, the flow being confined to $y>0$.The two equal and opposite forces are applied along the $x$-axis so that the wall is stretched keeping the origin fixed.

The steady incompressible two dimensional boundary layer equations for this visco-elastic second order fluid with heat and mass transfer in usual notation are

$$
\begin{aligned}
& \frac{\partial u}{\partial x}+\frac{\partial v}{\partial y}=0, \\
& u \frac{\partial u}{\partial x}+v \frac{\partial u}{\partial y}=v \frac{\partial^{2} u}{\partial y^{2}}-k\left\{\frac{\partial}{\partial x}\left(u \frac{\partial^{2} u}{\partial y^{2}}\right)+\frac{\partial u}{\partial y} \cdot \frac{\partial^{2} v}{\partial y^{2}}+v \cdot \frac{\partial^{3} u}{\partial y^{3}}\right\}-\frac{v}{k^{\prime}} u-\frac{\sigma \beta_{0}^{2} u}{\rho}
\end{aligned}
$$


where

$$
\begin{aligned}
& v=\frac{\mu}{\rho}, \quad k=-\frac{\alpha_{1}}{\rho}, \\
& u \frac{\partial T}{\partial x}+v \frac{\partial T}{\partial y}=\frac{v}{\operatorname{Pr}} \frac{\partial^{2} T}{\partial y^{2}} \\
& u \frac{\partial c}{\partial x}+v \frac{\partial c}{\partial y}=D \frac{\partial^{2} c}{\partial y^{2}} .
\end{aligned}
$$

The boundary conditions are

$$
\begin{array}{lll}
u=u_{w}=b_{x}, & v=0, & T=T_{w} \quad \text { at } \quad y=0, \\
u=0, & T=T_{\infty} \quad \text { at } \quad y=\infty .
\end{array}
$$

Here $u$ and $v$ are the velocity components along the $x$ and $y$ directions, respectively.

$T$ is the temperature, $k$ is the parameter associated with the visco-elastic fluid, Pr is the Prandtl number and $v$ is the kinemetic viscocity.

Introducing the stream function $\psi$ such that

$$
\begin{aligned}
& u=\frac{\partial \psi}{\partial y}, \quad v=-\frac{\partial \psi}{\partial x} \quad \text { and defining } \\
& \psi=\frac{\psi}{u_{0} h_{0}}=\frac{\bar{x}}{\sqrt{\operatorname{Re}}} f(\eta), \quad \theta(\eta)=\frac{T-T_{\infty}}{T_{W}-T_{\infty}} \quad \text { and } \quad \varphi(\eta)=\frac{C-C_{\infty}}{C_{w}-C_{\infty}}
\end{aligned}
$$

Equations (2.1) to (2.6) are transformed to

$$
\begin{aligned}
& f_{\eta}(\eta)^{2}-f(\eta) f_{\eta \eta}(\eta)=f_{\eta \eta \eta}(\eta)+ \\
& -k_{1}\left\{2 f_{\eta}(\eta) f_{\eta \eta \eta}(\eta)-f_{\eta \eta}^{2}(\eta)-f(\eta) f_{\eta \eta \eta \eta}(\eta)\right\}-\left(m+k_{2}\right) f(\eta) \text {, } \\
& \theta_{\eta \eta}(\eta)+\operatorname{Pr} f(\eta) \theta_{\eta}(\eta)=0 \\
& \varphi_{\eta \eta}(\eta)+\operatorname{Sc} f(\eta) \varphi_{\eta}(\eta)=0 \\
& f=0, \quad f_{\eta}=\theta=\varphi=1 \quad \text { at } \quad \eta=0, \\
& f_{\eta}=\theta=\varphi=0 \quad \text { at } \quad \eta=\infty \\
& \text { where } \quad \bar{x}=\frac{x}{L}, \quad \bar{y}=\frac{y}{L}, \quad u_{0}=c L, \quad k_{1}=\frac{K C}{v}, \quad \eta=\sqrt{\operatorname{Re}} Y .
\end{aligned}
$$

$\operatorname{Re}=L u_{0}$ is the Reynold's number, $L$ and $u_{0}$ are the characteristic length and velocity, respectively. The momentum Eq.(2.8) is uncoupled with the energy and concentration Eqs (2.9) and (2.10).

By differentiating Eq.(2.8) with respect to $\eta$ and applying the boundary conditions (2.11), one gets 


$$
f_{\eta \eta \eta}(\eta)=\frac{f_{\eta \eta}(\eta)\left(1+M+k_{2}\right)}{1-k_{1}},
$$

for $k_{1}=1$, the value of $f_{\eta \eta \eta \eta}$ at $\eta=0$ becomes infinity. So, the limit of applicability of the solution of the problem becomes $k_{1}<1$. It is important to note that Eqs (2.8) and (2.11) represent a two point non linear fourth order differential equation having only three boundary conditions. The fourth boundary condition is obtained by using Eq.(2.11) in Eq.(2.8)

$$
f_{\eta \eta \eta}(\eta)\left(1-2 k_{1}\right)+k_{1} f_{\eta \eta}^{2}=\left(1+M+k_{2}\right),
$$

at

$$
\eta=0 .
$$

It is interesting to mention that for $k_{1}=1 / 2$ the non-dimensional surface velocity gradient, $f_{\eta \eta}(0)$ from Eq.(2.14) is found to be

$$
\pm \sqrt{2\left(1+M+k_{2}\right)} .
$$

Because of the boundary condition (2.14), we can obtain the following two closed form solutions for Eqs (2.8) and (2.11)

$$
\begin{aligned}
& f_{1}(\eta)=a_{1}\left(1-e^{-\frac{\eta}{a_{1}}}\right), \\
& f_{2}(\eta)=a_{2}\left(1-b_{2} e^{-\zeta} \cos \zeta\right)
\end{aligned}
$$

where

$$
a_{1}=\sqrt{\frac{1-k_{1}}{1+M+k_{2}}} ; \quad a_{2}=\frac{1+M+k_{2}}{\sqrt{k_{1}}} ; \quad b_{2}=\frac{2}{3}\left\{\frac{1+\left(k_{1} / 1+M+k_{2}\right)^{3}}{1+\left(k_{1} / 1+M+k_{2}\right)}\right\}^{1 / 2} .
$$

The above solutions (2.15.1) and (2.15.2) are quite different solutions because

$$
f_{1 \eta \eta}(0)=-\sqrt{\frac{1+M+k_{2}}{1-k_{1}}}<0 \quad \text { and } \quad f_{2 \eta \eta}(0)=\frac{1-k_{1}}{\left(\frac{k_{1}}{1+M+k_{2}}\right)^{3 / 2}}>0 .
$$

For magnetic parameter and porous parameter, $M=k_{2}=0$, our solution reduces to that of Lawrence et al. (1992), Troy et al. (1987).

Further it has been proved simultaneously by McLeod and Rajagopal (1987) and Troy et al. (1987) that Eqs (2.8), (2.11) and (2.14) have a unique solution, as 


$$
f(\eta)=1-e^{-\eta} \quad \text { for } \quad k_{1}=0
$$

We assume the first solution (2.15.1) as a realistic solution of the problem because of the following reasons.

For small values of $k_{1}=0.01, M=1.0$ and $k_{2}=1.0$, the surface velocity gradient from the first solution is

$$
f_{l \eta \eta}(0)=-\sqrt{\frac{1+M+k_{2}}{1-k_{1}}}<0
$$

and its value from the second solution

$$
f_{2 \eta \eta}(0)=\frac{1-k_{1}}{\left(\frac{k_{1}}{1+M+k_{2}}\right)^{3 / 2}}>0 \quad \text { for } \quad k_{1}=0, \quad M=0, \quad k_{2}=0, \quad \text { is } \quad f^{\prime \prime}(0)=-1 \text {. }
$$

Such a major change cannot be expected in the value of $f_{\eta \eta}(0)$ for a small value of $k_{1}$ because a viscous fluid with slight elasticity produces a boundary layer only slightly altered in its dimensions from a viscous fluid since the first solution (2.15.1) gives insight into the boundary layer for weakly elastic fluids, in the sense that $k_{1}<<1$. It is the elastic solution for $0<k_{1}<1$.

\section{Heat and mass transfer analyses}

Since the thermal boundary layer and concentration boundary layer thickness are of the orders of $\frac{1}{\sqrt{\operatorname{Pr} \operatorname{Re}}}$ and $\frac{1}{\sqrt{S c \operatorname{Re}}}$, the transformation co-ordinate $\eta$ in the boundary layer equation for energy (2.9) and for concentration (Rajagopal et al., 1984) are further modified to

$$
\tau=\sqrt{\operatorname{Pr} \eta} \quad \text { and } \quad \chi=\sqrt{\operatorname{Sc} \eta} .
$$

Using relation (2.16), the boundary layer equations for energy and concentration can be written as

$$
\begin{aligned}
& \ddot{\theta}(\tau)+\sqrt{\operatorname{Pr}} f\left(\frac{\tau}{\sqrt{\operatorname{Pr}}}\right) \dot{\theta}(\tau)=0, \\
& \ddot{\varphi}(\chi)+\sqrt{\operatorname{Sc}} f\left(\frac{\chi}{\sqrt{\operatorname{Sc}}}\right) \dot{\varphi}(\chi)=0 .
\end{aligned}
$$

And the corresponding boundary conditions are

$$
\begin{array}{lllll}
\theta=1 \quad \text { at } \quad & \tau=0 \quad: \quad \theta=0 \quad \text { at } & \tau=\infty, \\
\varphi=1 \quad \text { at } \quad \chi=0 \quad: \quad \varphi=0 \quad \text { at } & \chi=\infty .
\end{array}
$$


Integrating Eqs (3.2) and (3.3) with respect to the boundary conditions (3.4) and (3.5) one obtains the solutions for the energy equation and diffusion equation as

$$
\begin{aligned}
& \theta=1+\dot{\theta}(0) \int_{0}^{\tau} e^{-F(\alpha)} d \alpha \\
& \varphi=1+\dot{\varphi}(0) \int_{0}^{\chi} e^{-G(\alpha)} d \alpha
\end{aligned}
$$

where $\quad F(\alpha)=\sqrt{\frac{\operatorname{Pr}\left(1-k_{1}\right)}{1+M+k_{2}}}\left\{\alpha-\sqrt{\operatorname{Pr}} f\left(\frac{\alpha}{\sqrt{\operatorname{Pr}}}\right)\right\}$,

$$
G(\alpha)=\sqrt{\frac{\operatorname{Sc}\left(1-k_{1}\right)}{1+M+k_{2}}}\left\{\alpha-\sqrt{\mathrm{Sc}} f\left(\frac{\alpha}{\sqrt{\mathrm{Sc}}}\right)\right\},
$$

and

$$
\begin{aligned}
& \dot{\theta}(0)=-\left\{\int_{0}^{\infty} e^{-F(\alpha)} d \alpha\right\}^{-1}, \\
& \dot{\varphi}(0)=-\left\{\int_{0}^{\infty} e^{-G(\alpha)} d \alpha\right\}^{-1} .
\end{aligned}
$$

\section{Skin friction}

The skin friction co-efficient $C_{f}$ is derived as follows

$$
C_{f}=\frac{\left.\mu \frac{\partial u}{\partial y}\right|_{y=0}}{\frac{1}{2} \rho u^{2}}=\frac{2}{\sqrt{\operatorname{Re} x}} f_{\eta \eta}(0) .
$$

\section{Nusselt number}

The heat transfer co-efficient can be derived as

$$
\mathrm{Nu}=\frac{-\left.k \frac{\partial T}{\partial y}\right|_{y=0}}{-k\left(T_{w}-T_{\infty}\right)}=-\sqrt{\operatorname{Pr} \operatorname{Re} \bar{x}} \cdot \dot{\theta}(0) .
$$

Here $\operatorname{Re} x=\operatorname{Re} \bar{x}, \rho$ is the density, $\mu$ is the dynamic viscosity and $k$ is the thermal conductivity. 


\section{Sherwood number}

The mass transfer co-efficient can be obtained as

$$
\mathrm{Sh}=\frac{-\left.D \frac{\partial c}{\partial y}\right|_{y=0}}{-D\left(C_{w}-C_{\infty}\right)}=-\sqrt{\operatorname{ScRe} \bar{x}} \dot{\varphi}(0)
$$

where $D$ is the mass diffusivity.

\section{Results and discussion}

The problem of heat transfer in a visco-elastic boundary layer flow past a stretching sheet is discussed in this study. Velocity profiles and temperature profiles are given in closed form. Mathematically two closed form solutions are possible for Walters' liquid momentum equation out of which the first solution given by Eq.(15.5) is considered to be a physically realistic solution for the specified values of the Prandtl number $(\mathrm{Pr})$, Schmidt number $(\mathrm{Sc})$, non-Newtonian parameter $\left(k_{1}\right)$, permeability parameter $\left(k_{2}\right)$, magnetic parameter $(M n)$, the heat transfer co-efficient viz. the Nusselt number $(\mathrm{Nu})$, the mass transfer co-efficient viz, the Shrewood number (Sh), velocity transfer co-efficient viz skin friction co-efficient $(\tau)$ and the nondimensional temperature distribution $(\theta)$, concentration distribution $(\phi)$ along the surface can be found from these figures by specifying Reynolds number Re. From Figs 3 and 4 we can observe that the magnitude of the velocity gradient $f^{\prime}(o)$ increases for increasing values of the permeability parameter and magnetic parameter.

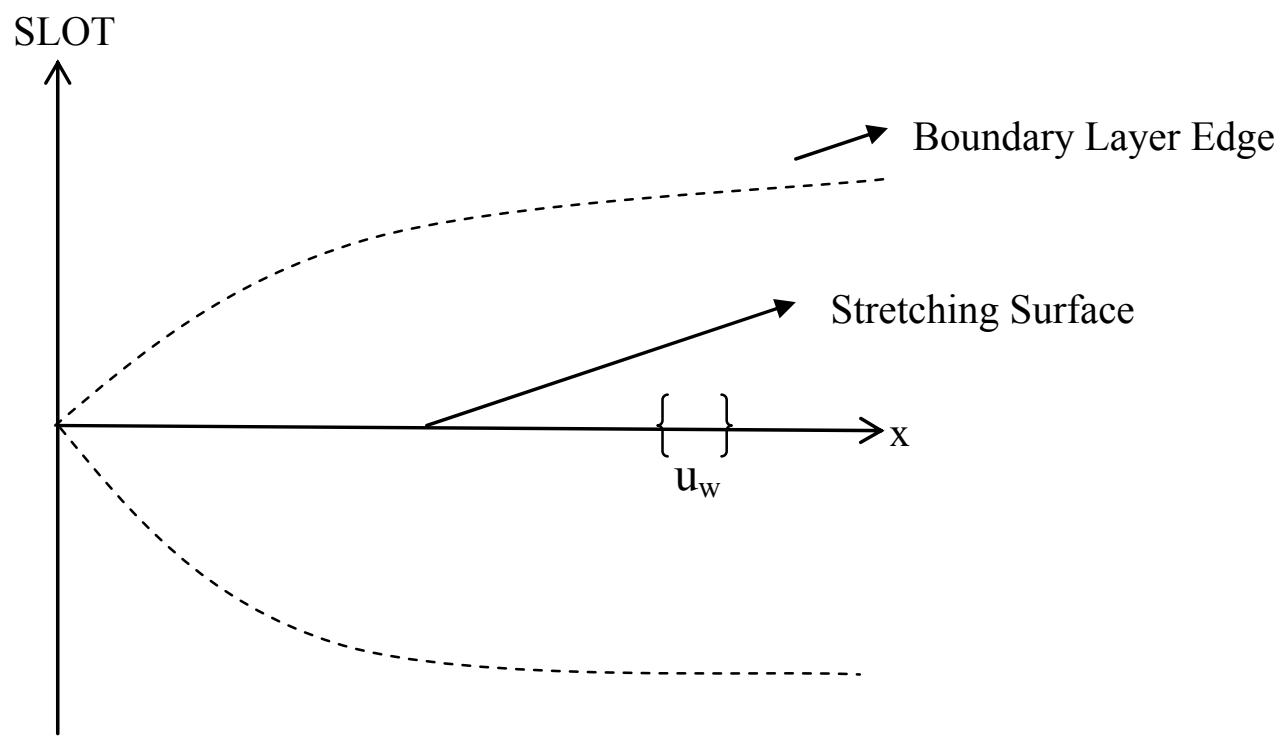

Fig.1. Visco-elastic fluid (Walter's fluid) flow over a stretching sheet. 


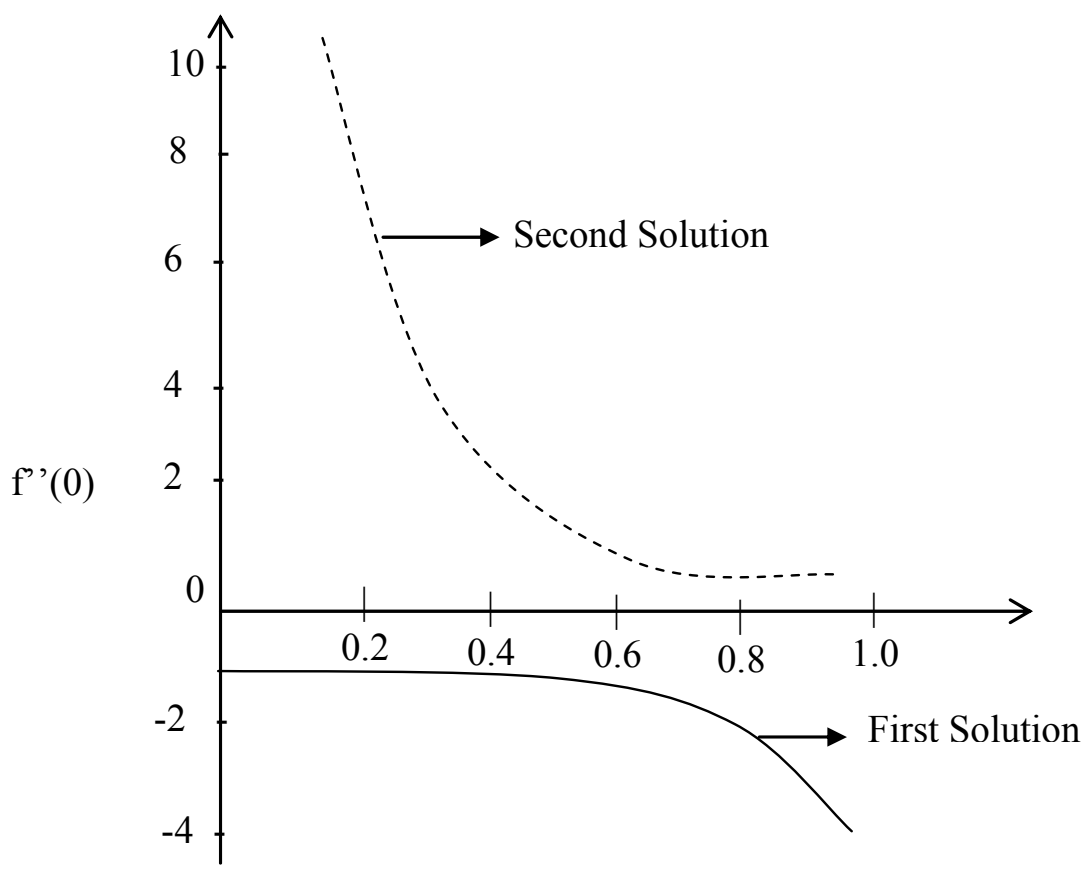

Fig.2. Surface velocity gradient $f^{\prime \prime}(0)$ for the first and second solution with non-Newtonian parameter $k_{l}$ for fixed values of permeability parameter $k_{2}=1$ and magnetic parameter $M_{n}=1$.

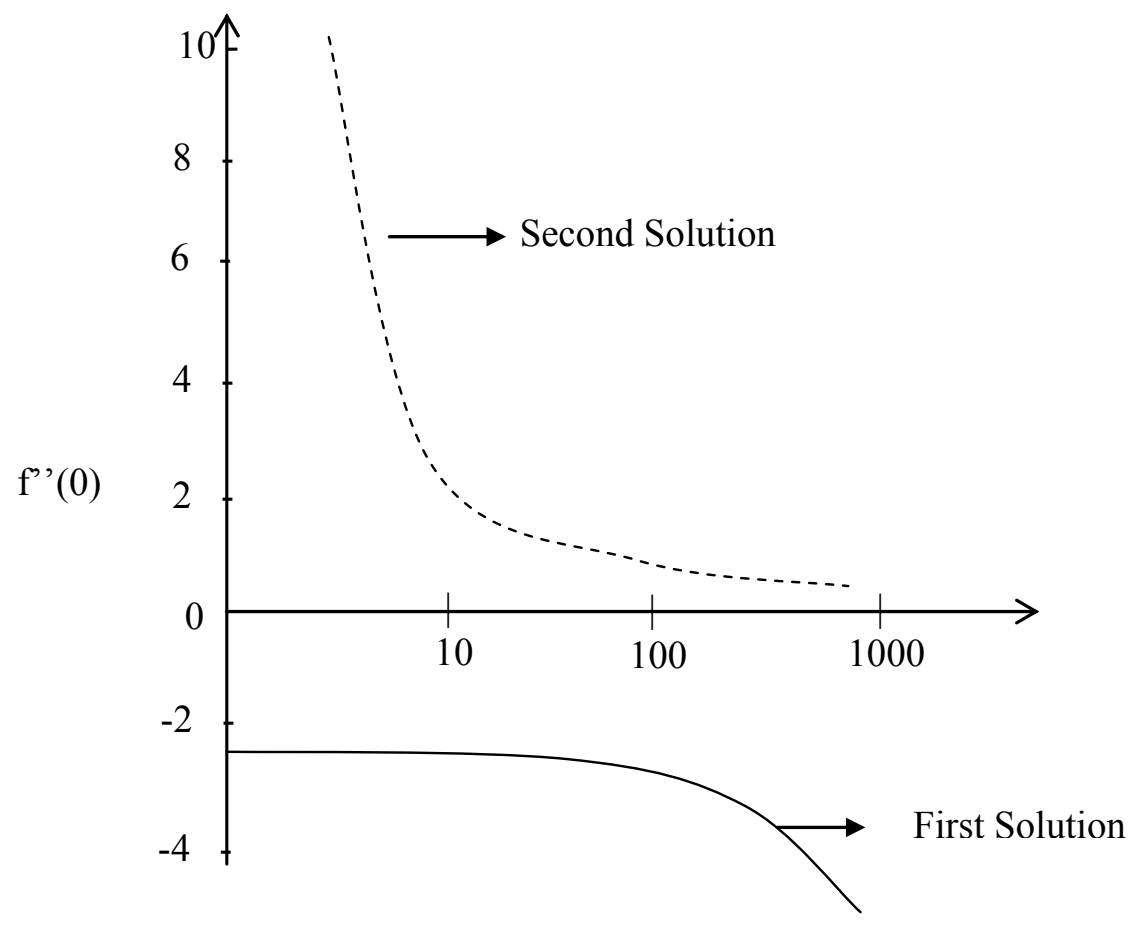

Fig.3. Velocity gradient $f^{\prime \prime}(0)$ for various values of permeability parameter $k_{2}=10,100,1000$ and for fixed values of non-Newtonian parameter $k_{1}=0.6$ and magnetic parameter $M_{n}=1$. 


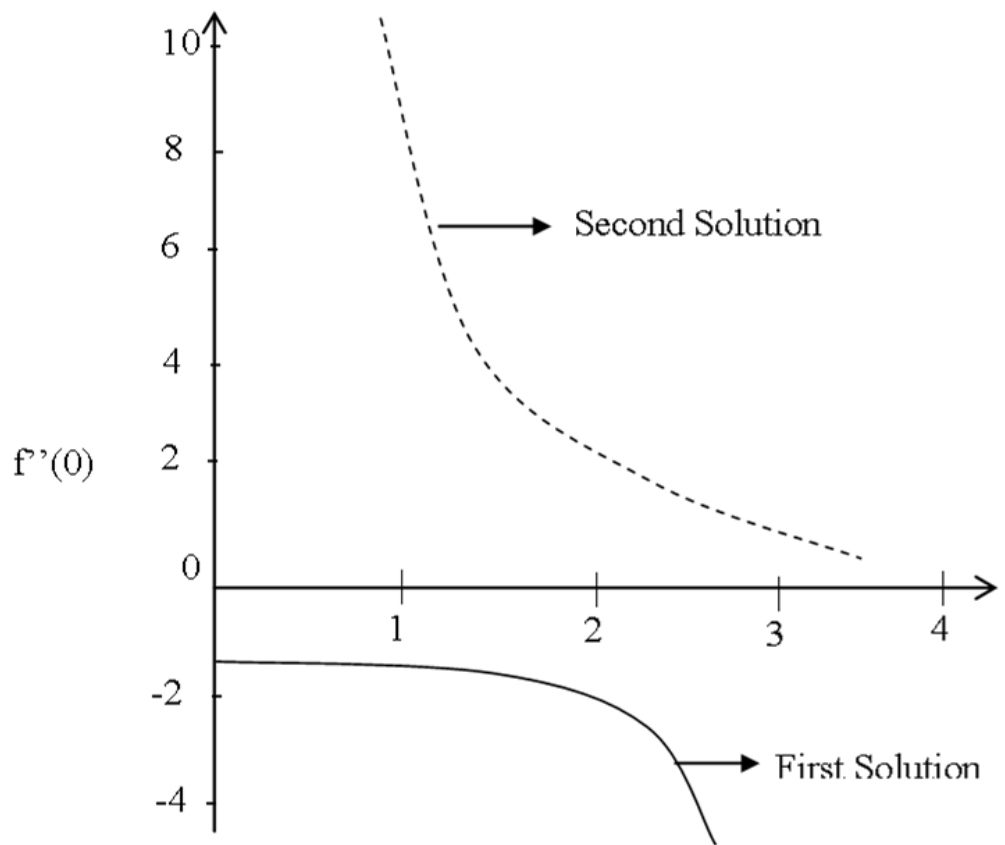

Fig.4. Surface velocity gradient with magnetic parameter $M_{n}=1,2,3$ for fixed values of permeability parameter $k_{2}=10$ and non-Newtonian parameter $k_{1}=0.6$.

Figures 5-7 depict the graphs of non-dimensional velocity profiles across the boundary layer for the non-Newtonian parameter $k_{1}=0.2,0.6$ and 0.8 and for fixed values of permeability and magnetic parameter $k_{2}=M=10$. It is found from the figures that the velocity boundary layer thickness decreases with increasing values of the non-Newtonian parameter $k_{l}$.

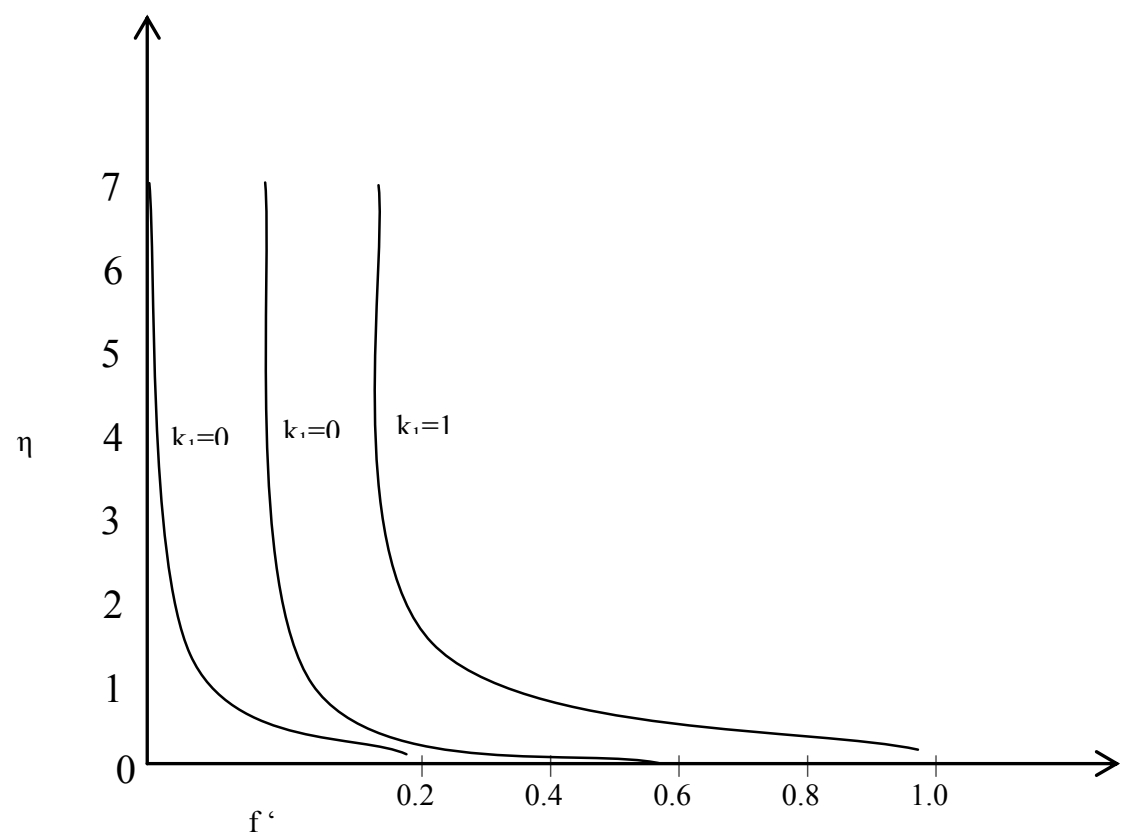

Fig.5. Velocity profiles for the non-Newtonian parameter $k_{1}=0.2,0.6,0.8,1.0$ when $k_{2}=10, M_{n}=10$.

In Figs 6 and 7 graphs of the non-dimensional temperature gradient and concentration gradients are drawn with the variation in the non-Newtonian parameter $k_{1}$. It is noted from Fig.6 that $\theta(0)$ decreases with increasing values of $k_{l}$ and the magnitude of $\theta(0)$ increases with the Prandtl number Pr. 
We found a negligible variation in the heat transfer co-efficient with respect to $k_{l}$ and for larger values of $\operatorname{Pr}>10$. As $\operatorname{Pr} \rightarrow \infty$, the magnitude of $\theta(0) \rightarrow \sqrt{2} / \pi$, which is independent of the visco-elastic parameter $k_{l}$.

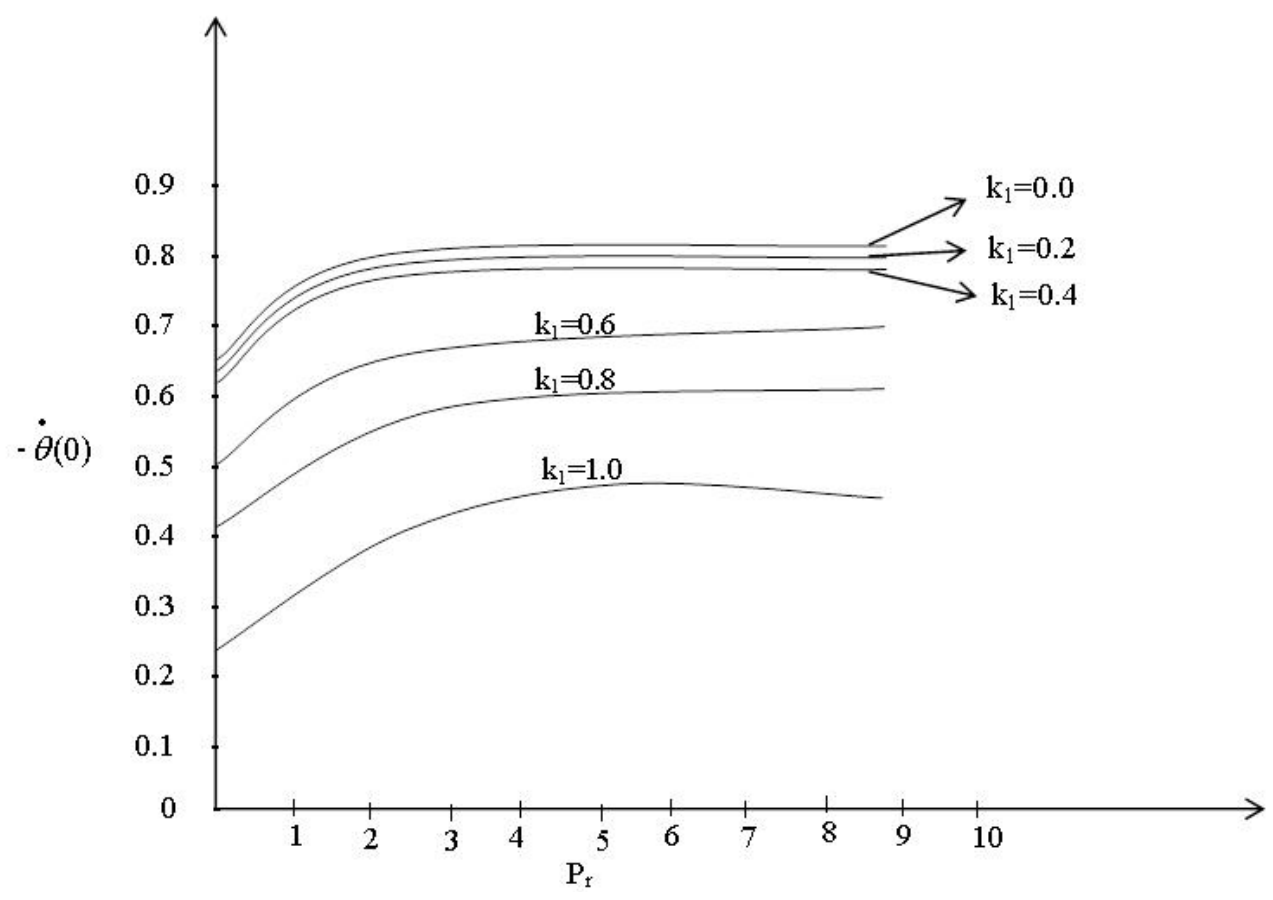

Fig.6. Surface temperature gradient $\dot{\theta}(0)$ with Prandtl number Pr and for various values of non-Newtonian parameter $k_{1}=0.0,0.2,0.4,0.6,0.8,1.0$.

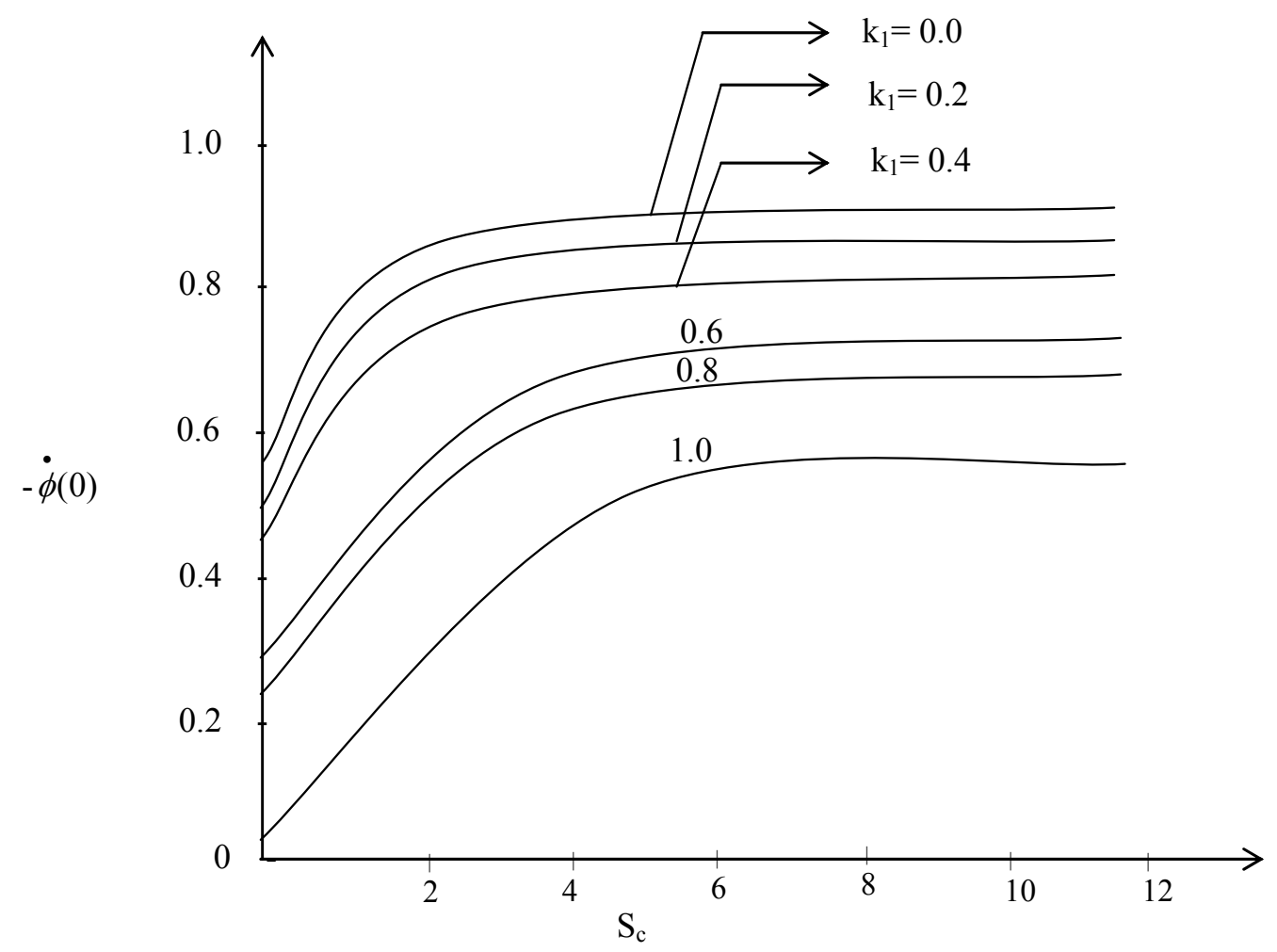

Fig.7. Wall concentration gradient with Schmidt number Sc and the non-Newtonian parameter $k_{l}$. 
From Fig.7 we noticed that the concentration gradient also decreases for an increase in the values of the visco-elastic parameter $k_{l}$ and there is a very negligible change in the mass transfer coefficient which is found for large values of the Schmidt number Sc.

Figures 8 and 9 present the non-dimensional temperature and concentration profiles across the boundary layer for the visco-elastic parameter $k_{1}=0.1,0.5$ and 0.9 and $\operatorname{Pr}=1$ to 10 and $\mathrm{Sc}=1$ to 10 . It is found from the figures that the thermal boundary layer thickness and the concentration boundary layer thickness increase with the visco-elastic parameter $k_{1}$.

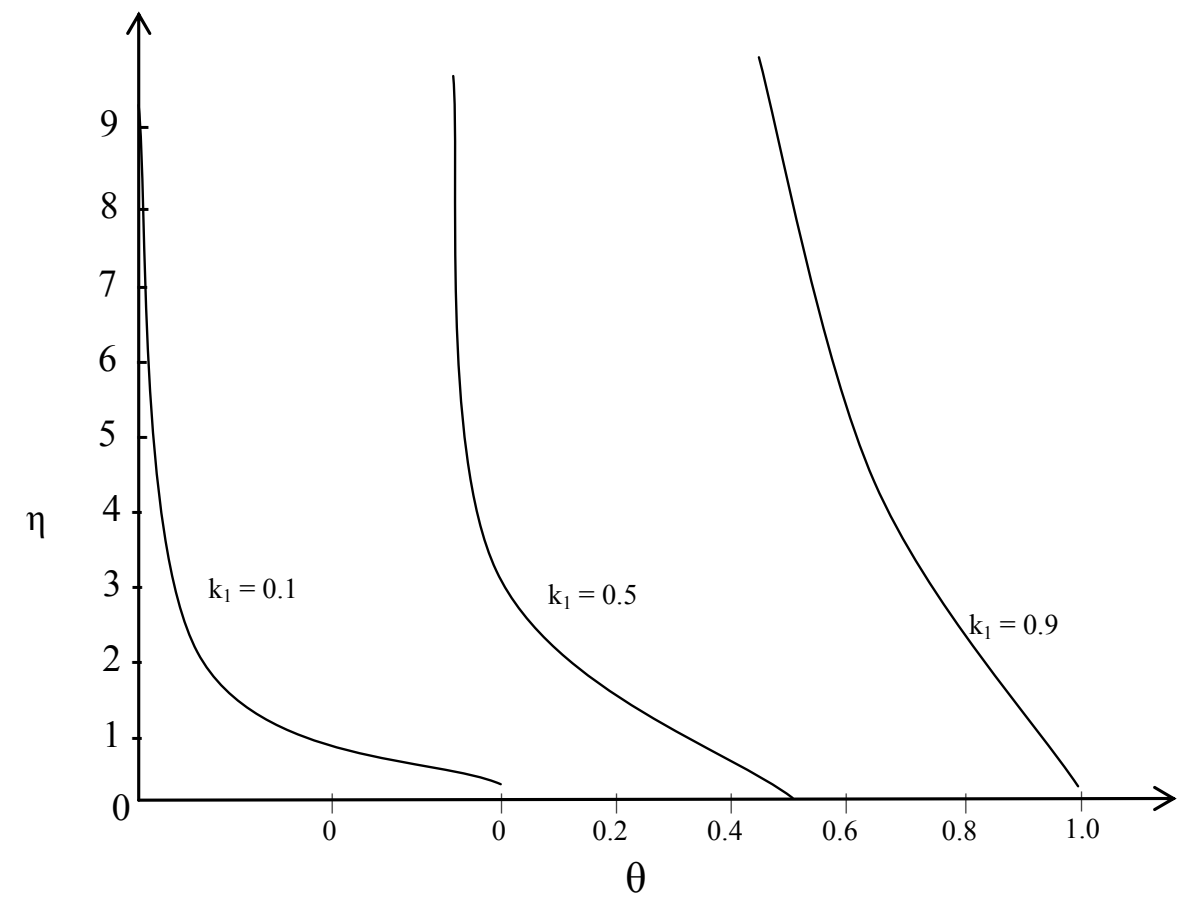

Fig.8. Temperature profiles for the non-Newtonian parameter $k_{1}=0.1,0.5,0.9$ and Prandtl number, $\operatorname{Pr}=0.7$.

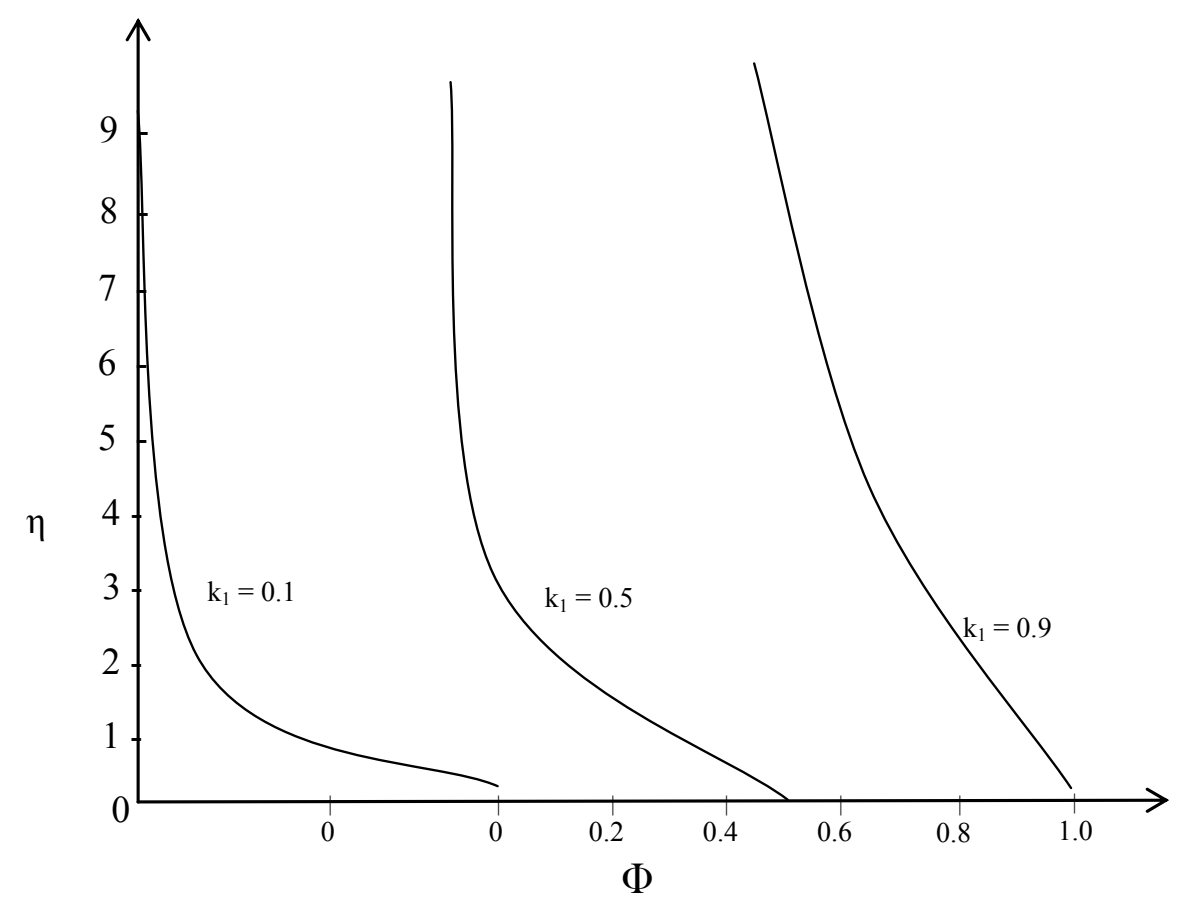

Fig.9. Concentration profiles for $k_{l}=0.1,0.5,0.9$, and Schmidt number $\mathrm{Sc}=2$. 


\section{Nomenclature}
$A$ - constant
$A_{1}$ - constant
$A_{2}$ - constant
$A_{3}$ - constant
$\boldsymbol{B}_{0}-$ magnetic induction
$B_{0}$ - magnetic field strength
$b$ - stretching rate
$C$ - species concentration
$C_{p}$ - specific heat capacity
$C_{w}$ - species concentration at the plate
$C_{\infty}$ - species concentration far away from the plate

$F_{x}, F_{y}$ - magnetic forces in $x$ and $y$ directions

$f$-dimensionless similarity variable

$\boldsymbol{H}$ - induced magnetic field

$\boldsymbol{H}_{0} \quad$ - applied magnetic field

$J_{w}$ - local wall heat flux per unit area

$k$ - thermal conductivity

$k^{\prime}$ - co-efficient of porosity

$k_{w}$ - conductivity of the fluid at the sheet

$k_{\infty}$ - conductivity of the fluid far away from the sheet

$k_{l}$ - visco-elastic parameter

$k_{2}$ - permeability parameter

$k_{0}$ - co-efficient of elastic velocity

$\ell$ - characteristic length

M - Kummer's function

$M n$ - magnetic parameter

$m_{w}-$ power law mass flux

$N(\tau)$ - distribution function of relaxation times

$\mathrm{Nu}_{\mathrm{x}}-$ Nusselt number

$n$ - order of the reaction

$P$ - pressure

Pr - Prandtl number

$p$ - hydrostatic pressure

$Q$ - heat source/sink

$q_{w}$ - local heat transfer rate per unit area

Re - Reynolds number

$r, s$ - wall temperature parameters

$S$ - non-dimensional parameter representing the relative magnitude of frequency to stretching rate

Sc - Schimdt number

$T_{w}$ - wall temperature

$T_{0}$ - on set temperature

$T_{\infty}$ - free stream temperature

$t$ - time

$t^{*}$ - dimensionless time

$u, v, w$ - velocity components along $x-, y$ - and $z$-axes respectively

$v$ - characteristic velocity

$v_{w}$ - suction velocity

$\alpha$ - root of momentum equation

$\beta$ - heat source/sink parameter

$\beta_{l}-$ reaction rate parameter 


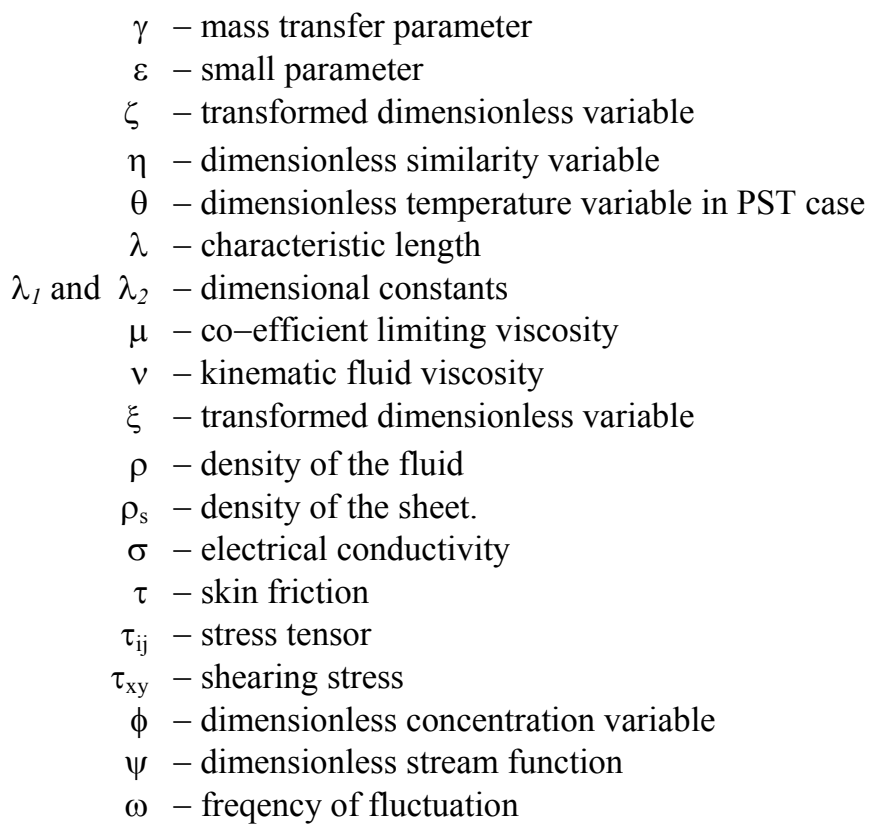

\section{Subscripts}

$w$ - with continuous surface condition

$\infty$ - ambient condition

\section{References}

Ahmad N., Patel G.S. and Siddappa B. (1990): Visco-elastic boundary layer flow past a stretching plate and heat transfer. - ZAMP, vol.41, pp.291.

Ashok V. Singh, Pallath Chandran and Nirmal C.S. (2000): Effect of transverse magnetic fluid on a flat plate thermometer. - Int. J. of Heat and Mass Transfer, vol.43, pp.3253.

Chaim T.C. (1982): Micropolar fluid flow over a stretching sheet. Journal of Applied Mathematic and Mechanic (ZAMM), vol.62, pp.565.

Chen C.K. and Char M.I. (1986): Heat transfer of a continuous stretching sheet with suction of blowing. - J. Math. Anal. Appl., vol.135, pp.568.

Crane L.J. (1970): Flow past a stretching plate. - ZAMP, vol.21, pp.645.

Dunn J.E. and Rajgopal K. (1995): Fluids of differential type- a critical review and thermodynamic analysis. - Int. Journal of Engg. Sci., vol.53, pp.689-729.

Fosdick R.L. and Rajapogal K.R. (1979): Anamolous features in the model of second order fluids. - Arch. Ration, Mech. Anal., vol.70, pp.145-152.

Gupta P.S. and Gupta A.S. (1977): Heat and mass transfer on a stretching sheet with suction or blowing. - Canad. J. Chem. Engg., vol.55, pp.744.

Khan S.K. and Sanjayanand E. (2005): Visco-elastic boundary layer flow and heat transfer over an exponential stretching velocity. - Int. J. of Heat and Mass Transfer, vol.48, p.811.

Layek G.C., Mukhopadhayay S. and Samad S.K.A. (2007): Heat and mass transfer analysis for boundary layer stagnation point flow towards a heated porous stretching sheet with heat absorption and suction or blowing. - Int. Comm. in Heat Mass Transfer, vol.34, pp.347.

Mcleod J.B. and Rajagopal K.R. (1987): On the uniqueness of flow of a Navier Stokes fluid due to stretching boundary. - Arch. Rat. Mech. Anal., vol.98, pp.385. 
Rajagopal K.R., Na T.Y. and Gupta A.S. (1984): Flow of a visco-elastic fluid over a stretching sheet. - Rheol Acta., vol.23, pp.213.

Rajagopal K.R., Na T.Y. and Gupta A.S. (1987): A non-linear boundary layer on a stretching sheet in a non-Newtonian fluid with uniform free stream. - J. Math. Phys. Sci., 21, pp.189.

Rajagopal K., Veena P.H. and Pravin V.K. (2008): Diffusion of chemically reactive species of an electrically conducting visco-elastic fluid immersed in a porous medium over a stretching sheet with prescribed surface concentration and prescribed wall mass flux. - IJAME, vol.13, No.2, pp.457-472.

Sakiadis B.C. (1964): Boundary layer behaviour on continuous solid surfaces. - AIChE Journal 7, pp.221.

Sam Lawrenee P. and Nageshwar Rao B. (1992): Heat transfer in the flow of visco-elastic fluid over a stretching sheet. - Acta Mechanica, vol.93, pp.53.

Sam Lawrenee P. and Nageshwar Rao B. (1993): Reinvestingation of the non-uniqueness of the flow of a visco-elastic fluid over a straight sheet. - QAM: Vol. LI - 1993 No.3, pp.401-404.

Siddappa B. and Subhas A. (1985): Non-Newtonian flow past a stretching plate. - ZAMP 36, pp.890.

Siddeshwar P.J. and Mahabaleshwar U.S. (2005): Effect of radiation and heat source on MHD flow of a visco-elastic liquid and heat transfer over a stretching sheet. - Int. J. Non-Linear Mechanics, vol.40, pp.807.

Subhas A. and Veena P.H. (1998): Visco-elastic fluid flow and heat transfer in a porous medium over a stretching sheet. - Int. J. Non-Linear Mech., vol.33, pp.531.

Surmadevi C.D. and Nath G. (1990): Flow and heat transfer of a visco-elastic fluid over a stretching sheet with variable wall temperature or heat flux. - Ind. J. Technology, vol.28, pp.93.

Takhar H.S. and Soundalgekar V.M. (1986): Flow and heat transfer of a micropolar fluid past a continuously moving porous plate. - Int. J. Heat Mass Transfer, vol.29, pp.196.

Thakar H.S. and Gorla R.S.R. (1991): Unsteady mixed convection boundary layer flow of a micropolar fluid near the lower stagnation point on a cylinder. - Int. J. Engg. Fluid Mech, vol.4, pp.337.

Tsou F.K., Sparrow E.M. and Goldstein R.J. (1967): Flow and heat transfer in the boundary layer on a continuous moving surface. - Int. J. Heat Mass Transfer, vol.10, pp.289.

Troy W.C., Overman H.E.A., Keener J.P. and Ermontrait G.B (1987): Uniqueness of flow of second grade fluid past a stretching sheet. - Journal of Quart. Appl. Math., vol.44, pp.753.

Veena P.H., Pravin V.K. and Nagabhooshan J.K. (2007): Unsteady mass transfer and magneto hydrodynamic flow of visco-elastic fluid over a stretching sheet saturated in a porous medium with suction/blowing. - IJAM E, 1, pp.263282.

Wen-Dong Chang (1989): The non-uniqueness of the flow of a visco-elastic fluid over a stretching sheet. - Quart. Appl. Math., vol.47, p.365. 